\title{
Maturation of pig oocytes in vivo and in vitro
}

\author{
R. Moor and Y. Dai* \\ The Babraham Institute, Babraham, Cambridge CB2 4AT, UK
}

In this review the concept that the origins of embryonic failure occur during oocyte development is explored. The four factors that determine oocyte viability, namely a normal growth phase, adequate follicle cell support during maturation, the completion of intracellular reprogramming before fertilization and the functioning of oocyte surveillance mechanisms, form the four sections of this review. The viability of pig oocytes at the end of the growth phase is compromised by presumptive spontaneous meiotic progression and by morphological heterogeneity. Determining the percentage and identity of viable dictyate oocytes, and identifying the reasons for the loss of viability, are key areas of future investigation. Although the requirement for follicle cell support during maturation is already established, little is yet known about the underlying signals and their transmission to the oocyte. The analysis of the action and nature of somatic signals will provide the foundation for further advances in the maturation of oocytes in vitro. Signalling cascades in oocytes control both the translation of masked mRNA and the modification and spatial localization of resultant proteins. The interdependent nature of this control system explains why inappropriate signals during maturation lead to subsequent embryonic mortality. Chromosomal errors during meiosis and early mitosis accumulate because of the leaky nature of the checkpoint system during the maternally regulated part of development: effective cell cycle surveillance is established only after the activation of the embryonic genome. In summary, we emphasize that the quality of the dictyate oocyte and the provision of appropriate signals in vitro are the principal determinants of maturational success.

\section{Introduction}

The female germ cell population is specialized because its numbers are fixed in prenatal life and decline progressively thereafter by either atresia or ovulation. Although the pig conforms to this pattern of germ cell behaviour, its cellular population of approximately $2 \times 10^{5}$ primordial follicles is larger than that in many other mammals (Cosden and Telfer, 1987). Despite this apparent advantage, only a small fraction of pig oocytes will develop into live offspring in the lifespan of the sow. Most of the oocyte population (>99\%) will be lost at

*Correspondence

Email: daiy@bbsrc.ac.uk 
various stages of oogenesis by the process of programmed cell death. As the loss of oocytes by atresia forms the subject of other contributions to this symposium it will not be discussed further in this review. Instead, we shall focus on the competence of the remaining oocytes, coupled with a consideration of the effectiveness of potential surveillance mechanisms that function in the maintenance of oocyte quality. The underpinning proposition is that the normal progression of early embryogenesis depends absolutely on a complex but ordered series of events during oogenesis. This protracted period of oocyte development includes an early mitotic phase of germ cell multiplication, the transition from mitosis to meiosis, meiotic arrest, genetic recombination, primordial cell inactivity, oocyte growth and a final culminating phase of intense cellular reorganization during maturation. The introduction of a haploid sperm nucleus at fertilization restores the diploid status of the egg and drives meiosis to its conclusion. The high incidence of early embryonic mortality in pigs indicates that this elaborate series of cellular events may often undergo aberrant changes that are not invariably detected by oocyte surveillance mechanisms (for reviews see Perry, 1954; Wilmut et al., 1986).

In addition to their normal development in vivo, oocytes from antral follicles have, over the past 20 years, been used for the in vitro production of embryos (for reviews see Hasler et al., 1995; Galli and Lazzari, 1996). Despite numerous studies in a variety of different animals, the percentage of offspring produced in vitro remains $<20 \%$. Although a significant part of the embryonic mortality associated with in vitro systems may be a result of the inherent quality of the oocytes, the remainder appears to be associated with imperfect in vitro technology. The causes of these imperfections can be ascribed broadly to three general deficiencies: there is an inadequate understanding of the intracellular processes underlying oocyte development, many studies contain a number of variables and many use inadequate methods of experimental assessment. For example, using measures of success such as fertilization, cleavage or cavitation are at best imperfect and often obscure subtle cellular abnormalities that contribute to later embryonic mortality. The concepts of limiting the number of experimental variables and using rigorous endpoints such as live births is of particular relevance to pigs, in which disappointingly few in vitro-produced embryos develop to term (Mattioli et al., 1989; Funahashi and Day, 1997; Kikuchi and Kashiwazaki, 1999).

The aim of this review is to emphasize deficiencies in our understanding of both the oocyte and the processes involved in its acquisition of developmental competence during maturation. In particular, the review will pose the following questions: to what extent are G2-stage pig oocytes in antral follicles developmentally compromised? What intrafollicular signals confer developmental capacity on oocytes and through what general mechanisms do the intracellular signals act? Finally, what surveillance mechanisms exist to detect defects in oocytes during their passage from germ cells to mature metaphase II oocytes? References to current in vitro protocols will be made only where such considerations illuminate significant issues about oocyte quality, intrafollicular signalling or cellular surveillance.

\section{Heterogeneity and developmental competence in oocyte populations}

\section{Heterogeneity and follicular dynamics}

It is axiomatic that heterogeneity is an essential feature of folliculogenesis in the ovaries of all mammals. Whether there are differences in the pool of primordial follicles is uncertain and raises the question of how and which follicles are recruited from this large reserve population into the growing classes. We postulate, but have not proven, that follicles differ even within the primordial pool. Regardless of the accuracy or otherwise of this hypothesis, there is no dispute about the importance of follicular heterogeneity once growth has been initiated. Pig 
follicles develop over a number of months from a structure composed of a single layer of flattened somatic cells to a complex multi-compartmental antral follicle in a highly ordered series of stages (Morbeck et al., 1994). Changes in the oocyte parallel these somatic cell developments. Genes are activated in a stage-specific manner; some of the resultant mRNA is translated immediately and the proteins are used to support the remarkable 200-fold increase in the volume of the developing oocyte. Other newly synthesized proteins are exported to form the zona pellucida or to act as signals between the growing oocyte and its associated follicle cells. However, not all the mRNA is translated and as the oocyte grows it accumulates large quantities of de-adenylated transcripts that are complexed with protein and stored in the cytoplasm (Gosden et al., 1995). It is this masked mRNA that will ultimately drive both the process of maturation and all the developmental events that occur before activation of the embryonic genome. Therefore, there can be no doubt that heterogeneity exists not only during all stages of folliculogenesis but is also an essential component of the ovarian regulatory system. However, should the oocyte be regarded as entering a homogeneous population once its growth phase is complete? Furthermore, if there are morphological differences between fully grown oocytes, can these be correlated with the subsequent developmental capacity of the egg?

\section{Heterogeneity within the fully grown dictyate oocyte population}

Knowledge about the inherent quality of oocytes removed from follicles by clinicians and research workers for the in vitro production of embryos is an important unresolved problem. Clearly, until this intractable question is resolved no definitive statements can be made about the absolute quality of either human or animal in vitro embryo production techniques. Questions about oocyte quality, especially in humans and cattle, have exercised workers for many years and have been the subject of a substantial number of papers all describing different methods that purport to identify healthy oocytes. However, with the exception of identifying grossly abnormal oocytes, no technique has yet been devised to distinguish oocytes with developmental competence from those that are inherently incapable of supporting development to term (Hyttel et al., 1997). Indirect evidence that a significant proportion of fully grown oocytes might be developmentally incompetent comes primarily from humans, in which approximately $20 \%$ of aspirated oocytes have been classified as morphologically degenerate (for review see Moor et al., 1998). Even when these oocytes are excluded, the number of babies produced from the remaining morphologically normal oocytes by in vitro maturation is still remarkably low. Moreover, genetic analyses on early cleavage stage embryos reveal a high incidence of chromosomal abnormality, indicating that many oocytes may be inherently genetically defective in humans. It is important to determine whether high percentages of genetically defective oocytes occur in other species also, or whether chromosomal abnormalities are predominantly a problem associated with the extended lifespan of humans.

In pigs, questions about oocyte homogeneity have focussed primarily on observed differences in the configuration of chromatin in the nucleus of fully grown oocytes and are based on a seminal paper by Motlik and Fulka (1976). These studies were carried out on the ovaries of 24 superovulated gilts that were examined at $4 \mathrm{~h}$ intervals after administration of hCG and from oocytes cultured in vitro. A total of 156 oocytes was examined before or within $4 \mathrm{~h}$ of hCG injection and $145(94 \%)$ exhibited a GVI type chromatin pattern: the remaining 11 oocytes displayed advanced stages of germinal vesicle breakdown (GVIV or early diakinesis). In contrast, the results of more recent studies using the same classification system indicate that an exceptionally low percentage (approximately $30 \%$ ) of oocytes collected from mediumsized follicles of prepuberal gilts or from sows in the luteal or early follicular phase remain in an 
inactive GVI state (see Funahashi and Day, 1997; Prather and Day, 1998; Guthrie and Garrett, 2000). Excluding possible differences in classification between the two groups of investigators, what could now account for this spectacular increase in the apparent spontaneous resumption of meiosis in pig oocytes from follicles 3-6 $\mathrm{mm}$ in diameter? The age of the pigs at death, the size and nature of the follicles selected for aspiration, their hormonal milieu and the composition of modern pig diets are among the possible explanations for the observed differences (Ding and Foxcroft, 1994; Hunter, 2000). Clearly, more research is needed on the causes of this presumptive precocious meiotic resumption. These studies could be preceded very profitably by an updating of the original follicle classifications using accurately staged oocytes taken directly from normal and synchronized pigs and three-dimensional nuclear reconstructions using modern confocal techniques.

It has been suggested that differences between GVI- and GVII-stage oocytes affect their subsequent maturation and development (Grupen and Nagashima, 1997; Prather and Day, 1998). To circumvent this, three approaches have been used to induce meiotic homogeneity before induction of the maturation process. The first approach has been to administer gonadotrophin (eCG) to gilts $72 \mathrm{~h}$ before oocyte collection. In the second approach, oocytes have been preincubated for $12 \mathrm{~h}$ without gonadotrophins. The third approach has been to expose oocytes to dibutyryl cyclic adenosine 3',5'-monophosphate (dbcAMP) or hypoxanthine to induce synchronization (Miyano et al., 1995; Funahashi and Day, 1997; Prather and Day, 1998). These meiotic synchronization protocols are of interest because they indicate that the GVI to GVII transition is readily reversible. However, both approaches also expose the oocytes to potentially damaging problems. The administration of gonadotrophins to ungulate oocytes can induce the premature unmasking of some mRNAs followed by precocious protein synthesis and a subsequent reduction in embryonic development (Moor et al., 1985). Equally, even the method of $\mathrm{LH}$ delivery in vivo appears to influence the subsequent developmental competence of oocytes in vivo (Oussaid et al., 1997). These experiments (Fig. 1) show that the administration of $\mathrm{LH}$ as a single bolus rather than in a pulsatile manner to sheep had no measurable effect on the completion of meiosis or on fertilization. However, the advantages of a pulsatile form of $\mathrm{LH}$ administration were not apparent until early development, when significantly more blastocysts $(P<0.01)$ were formed.

Taken together the above two studies indicate that exogenous hormone therapy can compromise egg quality and embryonic development. However, if used correctly gonadotrophins not only assist in the imposition of nuclear homogeneity (Funahashi and Day, 1997) but may also act to prime the oocyte before maturation. This postulated requirement for pre-maturation priming is considered by some investigators to occur in the period immediately preceding the release of $\mathrm{LH}$ and to be important for cytoplasmic maturation; prematuration priming plays no role in the regulation of the meiotic cell cycle (for review see Moor et al., 1998). There is some evidence of a small beneficial effect of pre-maturation priming in primates, cattle and also in pig oocytes, in which FSH priming increases development to the morula stage and beyond (Bolamba and Sirard, 2000).

In conclusion, it appears that the questions of oocyte viability may be complicated in pigs by problems associated with the spontaneous resumption of meiosis in oocytes from intermediate-sized follicles. This abnormality adds a further specialized challenge to the key problem of determining what percentage of fully grown oocytes is inherently capable of ultimately supporting development to term. Until this question is resolved, it will be impossible to be certain of the extent to which improvements can be made to our current methods of maturing oocytes in vitro. The resolution of this key question is likely to be expedited by the power of modern fluorescence-activated in situ hybridization (FISH) techniques for chromosome analysis (Munne et al., 1995; Harper and Delhanty, 1996). 

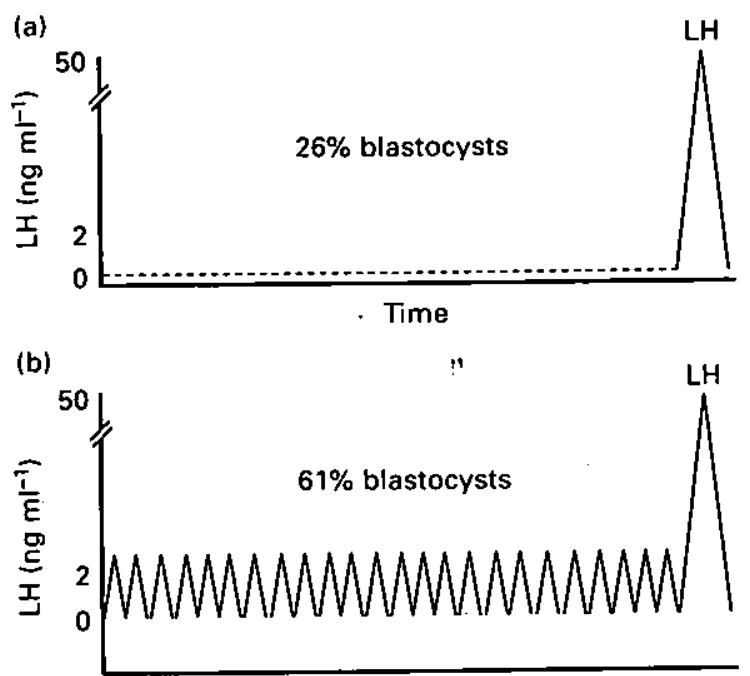

Time

(c)

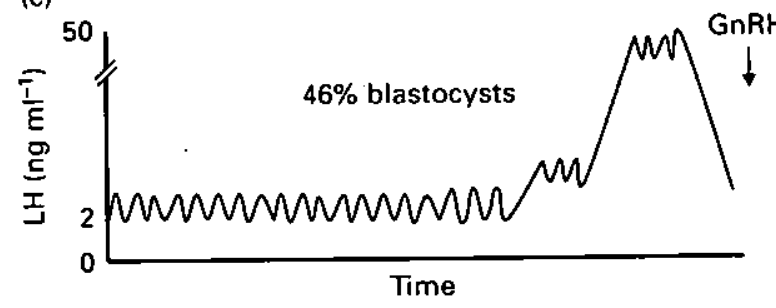

Fig. 1. An illustration that the mode of $\mathrm{LH}$ release in the $24 \mathrm{~h}$ preceding the $\mathrm{LH}$ surge in sheep (pre-maturation period) influences the subsequent developmental capacity of matured oocytes. (a) Inhibition of $\mathrm{GnRH}$. and, thus, LH pulsatility, was induced in the $24 \mathrm{~h}$ before maturation with the antagonist antarelix: oocyte maturation and ovulation were thereafter induced by a single injection of $\mathrm{LH}$. (b) Inhibition of natural $\mathrm{LH}$ pulsatility during the late follicular phase using antarelix. However, hourly injections of $\mathrm{LH}$ induced artificial pulsatility in the $24 \mathrm{~h}$ before a single ovulatory injection of $\mathrm{LH}$. (c) Untreated controls. Rates of blastocyst development were significantly higher in the groups of animals whose oocytes had been exposed to pulsatile $\mathrm{L} . \mathrm{H}$ administration in the $24 \mathrm{~h}$ before induction of maturation (graphs constructed from data presented by Oussaid et al., 1997).

\section{Follicular signals and intercellular communication}

The first requirement for the production of a viable egg is the growth and development of a competent dictyate oocyte and the second is for appropriate somatic cell support. A number of questions are raised by this assertion: is this support cell-type specific; what is the nature of the required support; when is it required; and by what pathways is the support delivered to the oocyte? 


\section{Follicular contribution to oocyte competence}

The question of whether and when follicle cell support is required during maturation is not new (for review see Moor and Warnes, 1978). Using in vivo approaches, Moor and Warnes (1978) showed that oocytes denied follicular support for the first third of the maturation period failed to develop into fetuses, although meiotic cycle progression was not inhibited. By linking these results with those derived from biochemical analyses of gene expression and cell fusion, it is now widely accepted that mammalian follicles generate inhibitory signals until after the LH surge (Dekel, 1996). Thereafter, positive follicular signals that are generated in the first third of maturation (inductive phase) initiate a phase of intracellular reprogramming that is completed in the second phase of maturation (cytoplasmic reprogramming phase). In addition to the provision of specialized signals for the completion of maturation, follicle cells also provide essential nutrient and metabolic support to the oocyte throughout the entire period of oocyte development. In the oocytes of domestic animals, this follicular cell support is required not only for growth but also for normal intracellular function, such as the maintenance of ionic balances in the cell and the maintenance of mRNA masking and stability (for reviews see Moor, 1983; Motlik and Fulka, 1986; Mattioli, 1994). Therefore, successful maturation of pig oocytes in vivo or in vitro depends on the presence of follicle cells able to generate and transmit the correct sequence of signals and support to induce and maintain the intracellular maturation programme.

\section{Signals and signalling pathways during maturation}

The oocyte membrane is remarkably poorly equipped to transport many of the metabolic products required by the oocyte (see Moor, 1983). Instead, substrates such as nucleotides, amino acids and phospholipids enter the oocyte cytoplasm through an extensive network of gap junctions. In pigs, cell coupling between the oocyte and the associated coronal cells is maintained throughout most of the maturation period. Its premature disruption in vitro does not affect meiotic progression but disrupts cytoplasmic maturation, fertilization and development totally (Mattioli et al., 1988; Mori et al., 2000). Although this loss of developmental potential is due in part to the intracellular depletion of essential molecules, junctional disruption also blocks the transmission of specific signals including those associated with calcium regulation and FSH stimulation (Fagbohun and Downs, 1991; Kaufman and Homa, 1993). The extent to which other intracellular regulators depend on junctional transport is unclear because of our lack of knowledge about the mode of action, or indeed even the nature, of many of the key signalling molecules. Studies on the somatic signals, their transmission and their intracellular action are likely to provide some of the more rewarding areas for future research on pig oocytes. These areas of study should include not only the somatic compartment but should also focus on receptors and electric potentials within the nuclear and cell membranes (Mattioli, 1994); this latter focus is important as membrane events affect polarity and cellular compartmentalization in amphibian, and probably also in mammalian, oocytes (see Berridge, 1988). A small number of examples will suffice to indicate both the complexity and the limits of our current knowledge on signalling during oocyte maturation. Oestrogen receptors have been located in oocytes (Wu et al., 1992) and oestrogen is also dominant in early preovulatory follicles. Inserting $17 \alpha$-hydroxylase inhibitors into ovine follicles in the early preovulatory phase subverts the normal steroid biosynthetic pathway. This change has no effect on meiotic progression but compromises early embryogenesis greatly by altering the synthesis of a small number of proteins in the oocyte (Osborn and Moor, 1983). Although oestrogens are important during the early phase of maturation, progesterone is required in the second phase of maturation to maintain junctional 
communication between the oocyte and the adjacent corona cells (Mattioli et al., 1988). Future analyses on steroid signalling will need to take into account the likelihood that the relationship between the oocyte and its steroid milieu is highly dynamic and may not even be of a direct nature. Likewise, growth factors and cytokines appear to have certain beneficial effects on oocytes matured in vitro but it is likely that the action of these might be on the follicle cells rather than directly on the oocyte. In addition to the above well-established signalling molecules, other more localized intrafollicular signalling systems may also be important. For example, after the íH surge, maturing oocytes secrete two major gelatinases and the associated follicle cells secrete the corresponding metalloproteinase tissue inhibitors (Brenner et al., 1989; Smith et al., 1999). The results of Funahashi et al. (1997) indicate that this interaction between oocyte matrix metalloproteinases (MMPs) and follicle cell inhibitors (tissue inhibitors of matrix metalloproteinases; TIMPS) may act as an activator during maturation (Fig. 2). Their results show firstly that TIMP acts during maturation only and is without effect if added after fertilization. Moreover, TIMP has no effect on cell cycle progression or fertilization but instead acts solely to induce a significant improvement in early development.

In conclusion, we believe that the unique contribution made by the follicle cells, often through intercellular communication, to oocyte development is clearly established. The intimacy of this oocyte-follicle interdependency is demonstrated graphically by the finding that the calcium stores of pig oocytes are rapidly depleted when they are removed from the follicle (Petr et al., 2001). Although it is apparent that a diverse range of somatic signals, ranging from inorganic ions to proteins, is involved in oocyte regulation, much remains to be discovered about the nature and action of both the inhibitory and stimulatory signalling systems. Moreover, it is apparent that many signals exert subtle effects that are not apparent until blastulation or beyond: this fact highlights the importance of using appropriate endpoints in signalling studies. Until both the full range of nutrients and instructive signals are identified, it will continue to be necessary to provide oocytes with appropriate signals by resorting to the inclusion of follicle cells in maturation systems (Staigmiller and Moor, 1984; Mattioli et al., 1989). However, the ultimate success of this cellular supplementation approach depends on the precision with which the culture system enables the follicle cells to mimic their counterparts in vivo. We consider that too little attention is given to this requirement in the design of in vitro maturation systems.

\section{Oocyte responses to intrafollicular signals}

The purpose of signals directed at oocytes in preovulatory follicles is to drive the two parallel programmes of meiotic progression and cytoplasmic reprogramming. These two programmes can, to a significant extent, occur independently of each other. However, it is only when both are synchronized that developmental competence is attained by the mature oocyte. Although the protein products required for each programme differ, the intracellular signalling pathways and their targets in the oocyte are comparable. In somatic cells, the ultimate role of most signals is either to regulate transcription or to alter the activity of the gene products by protein modification. In contrast, in fully grown oocytes the signalling pathways converge not on DNA regulation but on the mobilization and translation of masked $m R N A$, as virtually no transcription occurs in oocytes during maturation. Intracellular signals in oocytes are involved with the selection of appropriate stored transcripts for translation at precise times and in controlling the level and duration of translation. In addition to regulating translation, signals also act to control the activity of the resultant proteins by post-translational modification and to localize proteins to specific cellular compartments. Abnormalities in any of these regulatory steps may induce discontinuities in the maturation process with resultant failures during development. The 


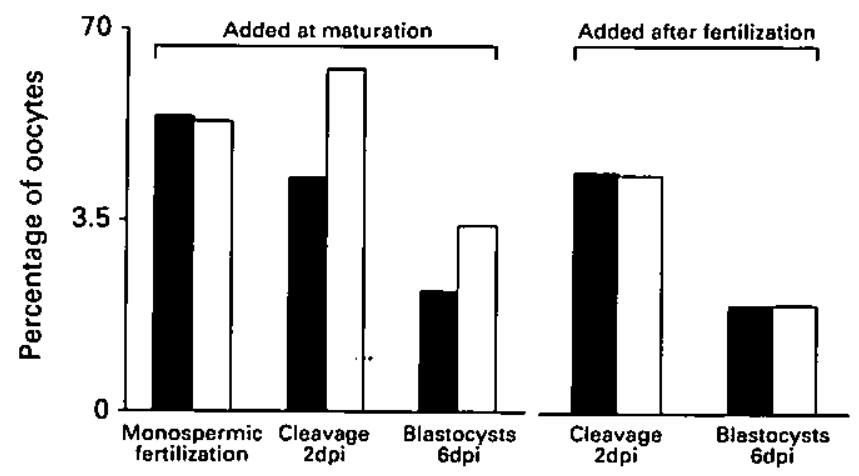

Fig. 2. Effect on early development of adding tissue inhibitor of metalloproteinase protein 1 (TIMP-1) to the medium used for maturation or culture of pig oocytes and embryos. $\mathbf{\square}$ : control; $\square$ : added TIMP-1; 2dpi: day 2 after implantation; 6dpi: day 6 after implantation. Addition of TIMP-1 during oocyte maturation enhanced embryo cleavage and blastulation. In contrast, addition of TIMP-1 during embryo culture had no effect on development (constructed from data published by Funahashi et al., 1997).

cytoplasmic reprogramming events controlled by stored mRNA extend to almost every component of the oocyte. The process of cytoplasmic reprogramming includes changes to the plasma membrane, changes to the position of intracellular organelles, synthesis of new products required for fertilization and early development, and the control of meiotic cycle events.

The progression of the meiotic cycle is unique because the nature of this form of cell division is entirely different from that in all other cells in the body. The complexity of the various specialized regulatory events that operate in maturing pig oocytes can be illustrated by reference to the events that occur during progress through the first meiotic M-phase. During this phase the $\mathrm{C} 2$-block is removed, chromatin condenses, the nuclear membrane disassembles, a spindle is formed, homologous chromosomes separate (while sister chromatids remain tightly adherent) and meiosis progresses from metaphase I to metaphase II with no intervening S-phase. The complexity and precision with which translational mechanisms control the availability of molecules involved in driving events that occur in the first M-phase are shown (Fig. 3).

The results presented (Fig. 3) highlight the fact that relevant mRNAs for meiosis are recruited at different times, for different durations and at different levels. Although the resultant new protein synthesis is essential for meiotic progression in pig oocytes, it is important to stress that not all controls are imposed on the translation process itself. Meiotic regulation includes both a temporal and spatial component. The time dimension is characterized by the synthesis and destruction of specific cell-cycle regulators at specific stages in meiosis. However, the modification and spatial localization of these molecules is equally important for the regulation of meiosis as the time of their synthesis (see Pines, 1999).

\section{Molecules controlling the first meiotic M-phase}

There is strong evidence that two tyrosine kinases, p34 cdc2 (MPF) and mitogen-activated protein kinase (MAPK), act in concert to induce G2- to $M$-phase progression in pig oocytes (Inoue et al., 1998; Motlik et al., 1998; Lee et al., 2000a). In the case of MPF kinase, the catalytic subunit ( $34^{\mathrm{cdc}}$ ) is stored in the oocyte as an inactive protein. The regulatory subunit 


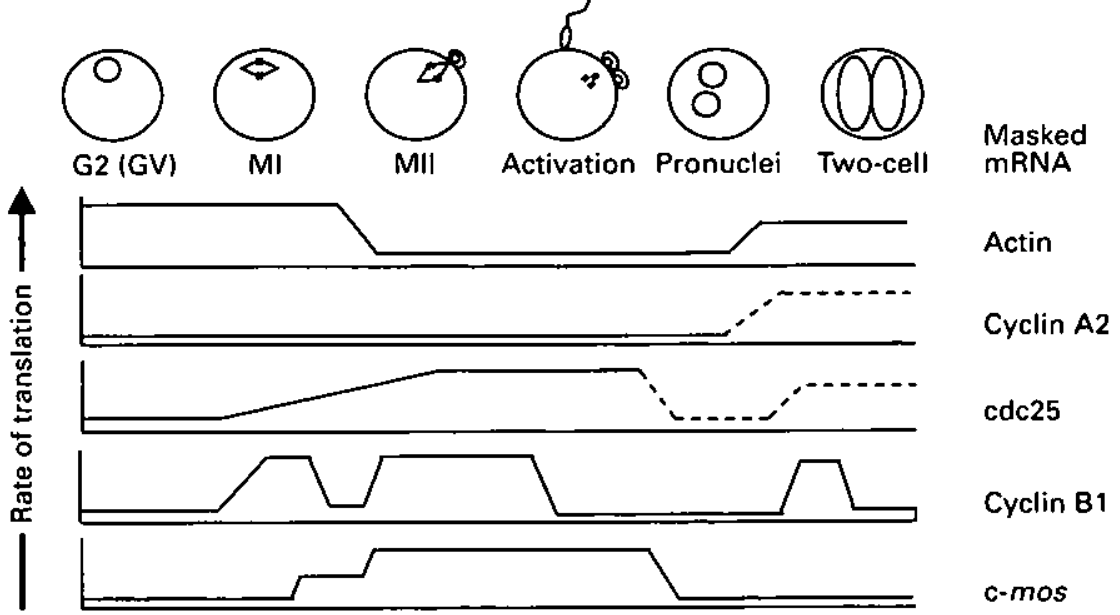

Fig. 3. The complex series of translational patterns generated by the mobilization of some of the masked mRNAs required to drive the cell cycle from the G2- (germinal vesicle) to the MII-stage of meiosis. The diagram illustrates the rate of translation of five species of mRNA plotted against the different stages of oocyte and egg development in pigs. Although the mRNAs shown represent a small fraction of the total pool of stored message only, nevertheless the unique nature of the translational pattern for each separate mRNA species is clearly shown.

(cyclin $\mathrm{B} 1$ ) requires de novo synthesis and complexing with the catalytic subunit during the late G2-phase. The MPF complex must then be activated by the removal of phosphates from Thr 14 and Tyr 15 of the p $34^{\text {cdc2 }}$ molecule before translocation to the nucleus, where it exerts many of its major effects. In contrast, the two MAPK kinases (ERK1 and ERK2) are stored as proteins in the cytoplasm of pig oocytes and become activated by phosphorylation and selective relocation (see Inoue et al., 1998). The MAPK cascade is important in many cell types, including pig oocytes, for the translocation of extrafollicular signals to their intracellular targets (Inoue et al., 1998). It is by examining the detail of how each translocational event, phosphorylation step and protein localization process is controlled that a full appreciation of the maturation process can be most easily gained. For example, the master cell cycle kinase regulator (MPF kinase) requires both the synthesis of B-type cyclin and dephosphorylation before it acquires enzyme activity in pig oocytes: the phosphatase required for MPF dephosphorylation is coded for by the cdc25 gene. The requirement for both cyclin B and cdc25 mRNA translation for entry into metaphase I in pig oocytes has been demonstrated by both direct mRNA injection (Fig. 4) and by antisense-mediated arrest of translation in dictyate oocytes (Dai et al., 2000). Thus, injection of mRNA coding only for either the B type cyclin (pig cyclin B1 or B2) or for the cdc25c protein on its own has only a limited effect on the induction of premature nuclear membrane breakdown (GVBD). In contrast, the injection of both cyclin B1 and cdc25 mRNA together induces premature GVBD in most oocytes (Y. Dai and R.M. Moor, unpublished).

Although both these results and those involving injection of antisense DNA (Dai et al., 2000 ) confirm that cdc 25 translation is required for GVBD, they provide no indication of how this translational event is regulated. Comparative studies on the pig cdc25 gene reveal that cdc25 mRNA in the oocyte differs from that in somatic cells. Moreover, these differences are retricted to the 3' untranslated region (3' UTR): oocyte-specific cdc25 mRNA contains a 400 


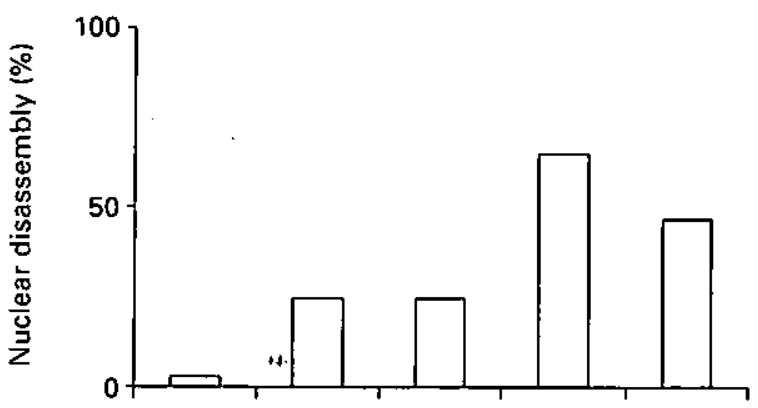

mRNA injection:

$\begin{array}{llllll}\operatorname{cdc} 25 & - & - & + & + & + \\ \text { Cyclin B1 } & - & + & - & + & - \\ \text { Cyclin B2 } & - & - & - & - & +\end{array}$

Fig. 4. Evidence that the synthesis of both a B-type cyclin and cdc25 protein is required for nuclear membrane breakdown (GVBD) in pig oocytes is provided by the microinjection of a dictyate (GV) oocyte with mRNA followed by confocal examination $15 \mathrm{~h}$ later $(n=30-54$ oocytes per group). Injection of either cyclin $\mathrm{B} 1$ or cdc25 mRNA alone induces premature nuclear membrane disassembly in $25 \%$ of oocytes. In contrast, injecting cdc25 and cyclin B1 together increases the percentage of premature nuclear membrane breakdown to $>65 \%$ (Y. Dai and R. M. Moor, unpublished).

nucleotide 3' UTR extension not found in any somatic cell (Newman and Dai, 1997). This unique segment of untranslated sequence contains the motifs that repress translation of the cdc25 mRNA throughout early growth and until the precise time in diakinesis when synthesis of cdc25 protein is required. At this point, RNA binding proteins, possibly modified by phosphorylation, bind to the cytoplasmic polyadenylation elements (CPEs) and the hexanucleotide polyadenylation signal within the extended 3' UTR of the stored cdc25 transcripts. The resultant RNA-protein interactions induce polyadenylation and the loading of the cdc25 mRNA onto polysomes for translation (Y. Dai, C. Lee and R. M. Moor, unpublished). By analogy with other systems it is probable, but has not been proven, that socalled zipcode proteins also bind to motifs in the extended 3' UTR to ensure the accurate localization of the proteins: The newly synthesized cdc25 protein requires phosphorylation before translocation to the nucleus, where it exerts its primary action on the MPF molecule (Hoffman et al., 1993; Dai et al., 2000). In the absence of cdc25 synthesis in pig oocytes, nuclear membrane disassembly (GVBD) in late diakenesis is prevented (Dai et al., 2000).

The example of cdc25 regulation shows that a range of phosphorylation cascades and translocation events is required to control the synthesis of this single phosphate. Inappropriate signals provided during in vitro maturation are likely to impair or distort one or more of the regulatory cascades that determine the correct temporal and spatial organisation of these events within the oocyte. Equally elaborate but entirely different sets of molecules are required both for the assembly of the spindle (Lee et al., 2000b) and for the accurate segregation of homologues at anaphase I (Y. Dai and R. M. Moor, unpublished). Aberrations in any of these processes, or in any of those that drive cytoplasmic maturation, generally also result in segregation errors, chromosomal imbalances or other developmental failures. 


\section{Checkpoint surveillance in pig oocytes}

Checkpoint mechanisms in somatic cells operate to eliminate errors during mitosis (Hartwell and Weinert, 1989). These mechanisms ensure that the progression of the cell cycle is errorfree; if errors are detected by the surveillance mechanisms then mitosis is arrested to enable the error to be corrected or the cell to enter the apoptotic pathway. Mitotic cells are blocked from entering $M$-phase if replication is incomplete or if the cells contain damaged DNA. Likewise, segregation of sister chromatids is prevented in $\mathrm{M}$-phase if abnormalities are detected in the spindle. Finally, replication is prevented in S-phase if segregation is incomplete (for review see Clarke and Gimenez-Abian, 2000). The question of what surveillance mechanism operates in oocytes to detect defects in meiosis I is particularly relevant for two reasons. Firstly, sister chromatids do not separate during the first meiotic $M$-phase, thereby raising important questions about the events monitored by meiotic checkpoints. Secondly, the high incidence of chromosomal non-disjunction associated with meiotic MI in humans (Angell, 1997) raises questions about the effectiveness of the entire surveillance system in oocytes.

Bradshaw et al. (1995) introduced an extensive set of lesions into dictyate oocytes immediately before maturation to determine whether checkpoints would prevent entry into M-phase. However, neither damaged DNA nor the introduction of unreplicated DNA into fully grown oocytes prevented chromatin condensation and nuclear membrane breakdown (Fulka et al., 2000). Although it is clear from studies on Drosophila (Roeder and Bailis, 2000) that checkpoints monitor the process of genetic recombination, there is no evidence that additional checkpoints monitor the integrity of DNA in fully grown dictyate oocytes of mammals. The search for meiotic $M$-phase checkpoints has been particularly intensive both because of the absence of checkpoints in dictyate oocytes and because of the unique chromosome segregation system that operates in the first meiotic metaphase. Cell fusion analyses, drug-induced spindle lesions, mutational targeting of DNA repair genes, DNA damage and genetically induced chromosomal imbalances have all been used in a variety of species, including the pig, to identify M-phase checkpoints. Using precisely staged mouse oocytes, Fulka et al. (1995) showed that the fusion of early metaphase I oocytes (before the capture of microtubules by kinetochores) to oocytes at later metaphase I stages invariably delayed the onset of chromosome segregation in the more advanced partner. The results of that study indicate that oocytes may emit 'wait' signals before kinetochore-microtubule attachment. These inhibitor signals disappear after kinetochore attachment and the chromosome segregation machinery is activated. This conclusion is supported by results from mice homologous for the targeted disruption of DNA mismatch repair genes (Woods and Hodge, 1999). However, other studies raise the possibility that the target for the sensing mechanism in the spindle checkpoint is dependent on microtubule tension rather than on kinetochore attachment itself (see Le-Maire-Adkins and Rouke, 1997; Yu and Muszynski, 1999). The presence of the spindle checkpoint is in contrast to the absence of a surveillance system for DNA damage during M-phase (Fulka et al., 2000).

In conclusion, these surveillance results indicate that despite the presence of checkpoints during recombination and in $\mathrm{M}$-phase, a surprisingly high proportion of mature human oocytes contain chromosomal errors (Hassold et al., 1996). As meiotic checkpoints appear leaky, it is a reasonable expectation that this would be compensated for by the presence of powerful checkpoints during early cleavage. Not only is this not the case but recent studies indicate that errors actually accumulate during early cleavage (Handyside and Delhanty, 1997; Munne and Cohen, 1998; Harrison and Kuo, 2000). Indeed, it appears possible that effective surveillance in embryos is not established until after activation of the embryonic genome at the mid-cleavage transition. 


\section{Future perspectives}

The development of pig embryos depends on three inter-linked processes, each dependent on the error-free completion of the preceding process. The first phase involving primordial cell selection and completion of growth offers the long-term potential for the induction of growth in vitro. Although in vitro growth would increase the number of fully grown oocytes available for maturation, this does not appear to be a high priority as exceptionally large numbers of pig ovaries are invariably available from commercially killed gilts. However, the development of methods for distinguishing between viable and developmentally compromised dictyate oocytes is a high priority. This methodology will be of central importance both in the selection of a homogeneous and viable population of oocytes for in vitro maturation and in the understanding of the link between oocyte quality and embryonic mortality in vivo. The problems of spontaneous resumption of meiosis in intermediate-sized antral follicles appear to be more acute in pig oocytes than in other species and this increases the concern about the quality of dictyate oocytes in pigs. Sensitive FISH methods of chromosome analysis, coupled with follicle dissection, now offer important opportunities for making significant progress in studies on the quality and heterogeneity of pig oocytes. However, it is possible that the elimination of genetically compromised oocytes will, on its own, be insufficient to ensure the optimal developmental potential of pig oocytes matured in vitro. A pre-maturation period of differentiation may be required before fully grown oocytes have the ability to respond to the full range of maturation signals.

The follicle cell compartment is our second recommended focus for future research on maturation. It is apparent that information on the nature, generation, transmission and action of intrafollicular signals is presently inadequate. Furthermore, it is entirely possible that the current expedient of adding follicular tissue to maturation systems in vitro might not provide the full range of signals to the oocyte. This is likely to be especially relevant if the maturation systems are not designed specifically to ensure normal follicle cell function. Modern methods of molecular analysis should now be used in studies on intrafollicular signalling systems. We anticipate that a systematic analysis of intrafollicular signals, coupled with methods of selecting and priming dictyate oocytes, will be central to the development of successful methods of in vitro maturation. However, success will be conditional on the use of rigorous experimental protocols and reliable biological endpoints for assessing factors that confer developmental competence on maturing oocytes.

The authors would like to thank L. Notton and K. Waterton for the artwork and for the preparation of the manuscript. Financial support from the BBSRC is gratefully acknowledged.

\section{References}

Angell R (1997) First-meiotic-division nondisjunction in human oocytes American Journal of Human Genetics 61 23-32

Berridge MJ (1988) Inositol lipids and calcium signalling Proceedings of the Royal Society of London (Biology) 234 359-378

Bolamba D and Sirard MA (2000) Ovulation and follicular growth in gonadotropin-treated gifts followed by in vitro fertilization and development of their oocytes Theriogenology 53 1421-1437

Bradshaw J, Jung T, Julka J, Ir and Moor RM (1995) UV irradiation of chromosomal DNA and its effect upon MPF and meiosis in mammalian oocytes Molecular Reproduction and Development 41 503-512

Brenner CA, Adler RR and Rappolee DA (1989) Genes for extracellular matrix-degrading metalloproteinases and their inhibitor, TIMP, are expressed during early mammalian development Genes and Development 3 848-859

Clarke DJ and Gimenez-Abian JF (2000) Checkpoints controlling mitosis Bioessays 22 351-363

Dai Y, Lee C, Hutchings A, Sun Y and Moor RM (2000) Selective requirements for $\mathrm{Cdc} 25 \mathrm{C}$ protein synthesis during meiotic progression in porcine oocytes Biology of Reproduction 62 519-532

Dekel $\mathbf{N}$ (1996) Protein phosphorylation/dephosphorylation in the meiotic cell cycle of mammalian oocytes Reviews of Reproduction 1 82-88

Ding I and Foxcroft GR (1994) Conditioned media produced by follicular shells of difíerent maturity affect maturation of pig oocytes Biology of Reproduction 50 1377-1384 
Fagbohun CF and Downs SM (1991) Metabolic and ligandstimulated meiotic maturation in the mouse oocytecumulus cell complex Biology of Reproduction 45 851-859

Fulka J, Ir, Moor RM and Fulka J (1995) Mouse oocyte maturation: meiotic checkpoints fournal of Experimental Cell Research 219 414-419

Fulka J, Jr, Tesarik J, Loi P and Moor RM (2000) Manipulating the human embryo: cell cycle checkpoint controls Cloning 2 1-7

Funahashi H and Day BN (1997) Advances in in vitro production of pig embryos Journal of Reproduction and Fertility Supplement 52 271-283

Funahashi H, McIntush EW, Smith MF and Day BN (1997) Effect of tissue inhibitor of metalloproteinase (TIMP-1) on early development of swine oocytes matured and fertilized in vitro. Theriogenology 47277 Abstract

Galli C and Lazzari G (1996) Practical aspects of IVMIVF in cattle Animal Reproduction Science 42 371-379

Gosden RG (1995) Oocyte development throughout life. In Cambridge Reviews in Human Reproduction, Gametes - The Oocyte pp 73-80 Eds JG Grudzinskas and $J$. Yovich. Cambridge University Press, Cambridge

Gosden RG and Telfer E (1987) Number of follicles and oocytes in mammalian ovaries and their allometric relationships Journal of Zoology 211 169-175

Grupen CG and Nagashima H (1997) Asynchronous meiotic progression in porcine oocytes matured in vitro: a cause of polyspermic fertilization? Reproduction, Fertility and Development 9 187-191

Guthrie HD and Garrett WM (2000) Changes in porcine oocyte germinal vesicle development as follicles approach preovulatory malurity Theriogenology 54 389-399

Handyside AH and Delhanty JD (1997) Preimplantation genelic diagnosis: strategies and surprises Trends in Genetics $13270-275$

Harper JC and Delhanty JD (1996) Delection of chromosomal abnormalities in human preimplantation embryos using FISH journal of Assisted Reproduction and Genetics 13 137-139

Harrison RH and Kuo HC (2000) Lack of cell cycle checkpoints in human cleavage stage embryos revealed by a clonal pattern oí chromosomal mosaicism analysed by sequential multicolour FISH Zygote 8 217-224

Hartwell LH and Weinert TA (1989) Checkpoints: controls that ensure the order of cell cycle events Science 246 629-634

Hasler JF, Henderson WB, Hurtgen PJ et al. (1995) Production, freezing and transier of bovine IVF embryos and subsequent calving results Theriogenology $\mathbf{4 3}$ 141-152

Hassold T and Abruzzo M (1996) Human aneuploidy: incidence, origin, and etiology Environmental and Molecular Mutagenesis 28 167-175

Hoffmann I, Clarke PR, Marcote MJ, Karsenti E and Draetta G (1993) Phosphorylation and activation of human cdc $25 \mathrm{C}$ by cdc2-cyclin B and its involvement in the selfamplification of MPF at mitosis EMBO fournal $1253-63$

Munter MG (2000) Oocyte maturation and ovum quality in pigs Reviews of Reproduction 5 122-130
Hyttel P, Fair T, Callesen H and Greve T (1997) Oocyte growth, capacitation and final maturation in cattle Theriogenology 47 23-32

Inoue M, Naito K, Nakayama T and Sato E (1998) Mitogenactivated protein kinase translocates into the germinal vesicle and induces germinal vesicle breakdown in porcine oocytes Biology of Reproduction 58 130-136

Kaufman ML and Homa ST (1993) Defining a role for calcium in the resumption and progression of meiosis in the pig oocytes Journal of Experimental Zoology $\mathbf{2 6 5}$ 69-76

Kikuchi K and Kashiwazaki N (1999) Developmental competence after transfer to recipients, of porcine oocytes matured, fertilized and cultured in vitro. Biology of Reproduction 60 336-340

Lee J, Miyano T and Moor RM (2000a) Localisation of phosphorylated MAP kinase during the transition from meiosis I to meiosis II in pig oocytes Zygote 8 119-125

Lee J, Miyano T and Moor RM (2000b) Spindle formation and dynamics of $\gamma$-tubulin and nuclear mitotic apparatus protein distribution during meiosis in pig and mouse oocytes Biology of Reproduction $621184-1192$

LeMaire-Adkins R and Radke K (1997) Lack of checkpoinı contral at the metaphase/anaphase transition: a mechanism of meiotic nondisjunction in mammalian fermales fournal of Cell Biology 139 1611-1619

Mattioli M (1994) Transduction mechanisms for gonadotrophin-induced oocyte maturation in mammals Zygote 2 347-349

Mattioli M, Galeati G, Bacci ML and Seren E (1988) Follicular factors influence oocyte fertilizability by modulating the intercellular co-operation between cumulus cells and oocytes Gamete Research 21 223-232

Mattioli M, Galeati G, Bacci ML and Seren E (1989) Developmental competence of pig oocytes matured and fertilized in vitro. Theriogenology 31 1201-1207

Miyano T, Ebihara M, Goto Y, Hirao Y, Nagai T and Kato S (1995) Inhibitory action of hypoxananthine on meiotic resumption of denuded pig follicular oocytes in vitro. Journal of Experimental Zoology 273 70-75

Moor RM (1983) Contact, signalling and cooperation between follicle cells and dictyate oocytes in mammals. In Current Problems in Germ Cell Differentiation pp 307-326 Eds A McLaren and CC Wylie. Cambridge University Press, Cambridge

Moor RM and Warnes GM (1978) Regulation of oocyte maturation in mammals. In Control of Ovulation $\mathrm{pp}$ 159-176 Eds DB Crighton, GR Foxcroft, NB Haynes and GE Lamming. Butterworths, London

Moor RM, Osborn JC and Crosby IM (1985) Gonadotrophin-induced abnormalities in sheep oocytes aiter superovulation Journal of Reproduction and Fertility 74 167-172

Moor RM, Dai Y, Lee C and Fulka J, Jr (1998) Oocyte maturation and embryonic failure Human Reproduction 423-236

Morbeck DE, Esbenshade KL, Flowers WL and Britt JH (1994) Kinetics of follicle growth in the prepubertal gilt Biology of Reproduction 47 485-491

Mori T, Amano T and Shimizu H (2000) Roles of gap junctional communication of cumulus cells in 
cytoplasmic maturation of porcine oocytes cultured in vitro. Biology of Reproduction 62 913-919

Motlik J and Fulka J (1976) Breakdown of the germinal vesicle in pig oocytes in vivo and in vitro. Journal of Experimemal Zoology 198 155-162

Motlik J and Fulka I (1986) Factors affecting meiotic competence in pig oocytes Theriogenology 25 87-96

Motlik J, Pavlok A, Kubelka M, Kalous J and Kala P (1998) Interplay between $\mathrm{CDC} 2$ kinase and map kinase pathway during maturation of mammalian oocytes Theriogenology 49 461-469

Munne S and Cohen J (1998) Chromosome abnormalities in human embryos Human Reproduction Update 4 842-855

Munne S, Alikani M, Tomkin C, Grifo J and Cohen J (1995) Embryo morphology, developmental rates and maternal age are correlated with chromosomal abnormalities Fertility and Sterility 64 382-391

Newman B and Dai $Y$ (1997) Transcription of c-mos protooncogene in the pig involves both tissue-specific promoters and alternalive polyadenylation sites Molecular Reproduction and Development 44 275-288

Osborn JC and Moor RM (1983) The role of steroid signals in the maturation of mammalian oocytes fournal of Steroid Biochemistry 19 133-137

Oussaid B, Mariana JC and Poulin N (1997) Effecl of LH pulses during the follicular phase on developmental competence of sheep oocytes 13th Scientific Meeting, European Embryo Transier Association Edition Fondation Marcel Mericux, Lyon 190

Perry IS (1954) Fecundity and embryonic montality in pigs Journal of Embryology and Experimental Morphology 2 308-322

Petr J, Rozinek I, Hruban V, Jilek F, Sedmikova $M$, Vanourkova $Z$ and Nemecek $Z$ (2001) Ultrastructural localization of calcium deposits during in vitro culture of pig oocytes Molecular Reproduction and Development 58 196-204

Pines J (1999) Four dimensional control of the cell cycle Nature Cell Biology 1 73-79

Prather RS and Day BN (1998) Practical considerations for the in vitro production of pig embryos Theriogenology 49 23-32

Roeder CS and Bailis JM (2000) The pachytene checkpoint Trends in Genetics 16 395-403

Smith MF, McIntush EW, Ricke WA, Kojima FN and Smith GW (1999) Regulation of ovarian extracellular matrix remodelling by metalloproteinases and their tissue inhibitors: effects on follicular development ovulation and luteal function Journal of Reproduction and Fertility Supplement 54 367-381

Staigmiller RB and Moor RM (1984) Effect of iollicle cells on the maturation and developmental competence of ovine oocytes matured outside the follicle Gametc Research 9 221-229

Wilmut I, Sales DI and Ashworth C) (1986) Maternal and embryonic factors associated with prenatal loss in mammals fournal of Reproduction and Fertility 76 $851-864$

Woods LM and Hodges CA (1999) Chromosomal iníluence on meiotic spindle assembly: abnormal meiosis $I$ in female MIh1 mulant mice Journal of Cell Biology 145 1395-1406

Wu T, Wang L and Wan Y (1992) Expression of estrogenreceptor gene in mouse oocyte and during embryogenesis Molecular Reproduction and Development $33407-412$

Yu HG and Muszynski MG (1999) The maize homologue of the cell cycle checkpoint protein MAD2 reveals kinetochore substructure and contrasting mitotic and meiotic localization patterns Journal of Cell Biology 145 $425-435$ 